4. Kuncevich V.M. Sintez robastno - optimal'nyh sistem upravlenija nestacionarnymi ob\#ektami pri ogranichennyh vozmushhenijah / V.M. Kuncevich // Problemy upravlenija i informatiki. — K., 2004. — №2 — S.19-31.

5. Timchenko V.L. Modelirovanie processov stabilizacii morskogo podvizhnogo ob\#ekta v rezhime dinamicheskogo pozicionirovanija / V.L. Timchenko, O.A. Uhin // Problemy upravlenija i informatiki. — Kiev, 2014. - № 4. - S. 77-88.

6. Timchenko, V.L. Robastnaja stabilizacija morskih podvizhnyh ob\#ektov na osnove sistem s peremennoj strukturoj obratnyh svjazej / V. L. Timchenko, Ju. P. Kondratenko // Problemy upravlenija i informatiki. - K., 2011. - № 3 S. 79-92.

\title{
ОПТИМИЗАЦИЯ ЗАГРУЗКИ ПТЛ ЭЛЕВАТОРОВ ЗЕРНОМ НА ОСНОВЕ САУ С КОММУТИРУЕМОЙ СТРУКТУРОЙ
}

Optimization of loading grain elevators PTL based ACS with a switched structure

Шестопалов С.В. ${ }^{1}$, Хобин В.А. ${ }^{2}$

${ }^{2}$ Одесская национальная академия пищевых технологий, г. Одесса

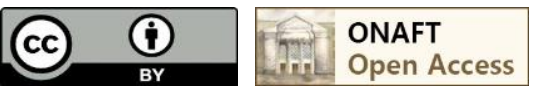

DOI: $10.15673 /$

\section{Аннотация}

Логистика движения зерна от производителя к потребителю - это логистика его перемещения между элеваторами различного назначения и объема хранения. Практика показывает, что в среднем в этой логистической цепи задействованы три - четыре элеватора. Основным и достаточно энергоемким процессом для всех элеваторов является процесс его перемещения поточно-транспортными линиями (ПТЛ), осуществляемый при приемке, подработке и отгрузке. Таким образом, объемы перемещаемого ПТЛ элеваторов зерна вцелом на порядок превосходят объемы его производства. На Украине это десятки миллионов тонн зерна ежегодно. Это обуславливает актуальность задачи снижения времени и удельных энергозатрат на эти перемещения. В ПТЛ входят группы последовательно включенных конвейеров различных типов для горизонтального и вертикального (нории) перемещения зерна. В системах автоматического управления (САУ) ПТЛ, и в прошлом веке и сейчас, реализовывались только функции сблокированных пуска и останова конвейеров, контроля возникновения аварийных ситуаций и предотвращения перерастания их в аварию, в частности путем аварийного отключения ПТЛ. Функция управления загрузкой ПТЛ, в т.ч. - ее оптимизации по критерию максимальной производительности и энергоэффективности, из-за сложности их свойств как объекта управления (ОУ), всегда сохранялась за человеком-оператором, как и ответственность за результаты неэффективного управления. Эта сложность определяется наличием у объекта управления совокупности специфических особенностей. К ним относятся: ограничения типа «аварийная ситуация» на режимные переменные процесса транспортирования; априорная неизвестность значений этих границ; невозможность прямого измерения некоторых важных режимных переменных; существенные изменения динамических свойств ОУ по каналам управления при изменении маршрута транспортирования; а также, и это принципиально важно, то, что оптимальные режимы работы ПТЛ приближаются к аварийным. Очевидно, что обеспечить работу ПТЛ в таких режимах возможно только при создании эффективных САУ, учитывающих все особенности ОУ. В статье подробно рассматривается алгоритм управления САУ с коммутируемой структурой, который учитывает перечисленные особенности ОУ, и обеспечивает работу ПТЛ с максимально достижимой производительностью и энергоэффективностью при гарантированном предотвращении аварийных ситуаций и аварийных остановов ПТЛ. Алгоритм управления отлажен в ходе процедуры имитационного моделирования, а САУ с коммутируемой структурой прошла производственные испытания на элеваторе. 


\section{Abstract}

Grain transferring logistics from producer to consumer is its transferring logistics between elevators of different purposes and different holding volumes. Experience shows that three-four elevators are used in this logistics chain. The main and the most energy-consuming process for all these elevators is the process of moving grain using threadtransport lines (TTLs) which takes place during taking over, undermining and discharge. Thereby, the volumes of grain transferred by elevator TTLs by far exceed the production volumes. In Ukraine this is tens of millions of tonns every year. This stipulates the actuality of the task of lowering time and energy consumings for these transfers. Different consecutively connected conveyors for horizontal and vertical (bucket chains) grain transfer are used in TTls. In systems of automatic control (SAC) for TTLs, in the last century and now, only were used functions of conveyors start and stop interlockings, control for incident occurrence and prevention from developing incident to breakdown, in particular, by emergency trips of TTLs. TTL load control function, including its optimization using criterion of maximum productivity and energy-efficiency, taking into accordance its specifics as control object (CO), always was delegated to human-operator as well as responsibility for the results of ineffective control. This complexity is determined by control object's different specific characteristics. These include: the restriction of «alarm status» type for mode variables of transferring process; ex ante uncertainty of these restrictions' values; impossibility of direct measurement of some important mode variables; substantial changes in CO dynamic characteristics in control channel when changing route of transfer; and also, and this is very important on principle, the fact that optimal modes of operation of TTLs are getting close to emergency modes. Obviously, only creation of effective SACs can provide TTLs' work in these conditions, and they also need to take into account different specifics of CO. Control algorithm of SAC with commutating structure, which take into account abovementioned CO specifics and provide TTLs work with maximum productivity and energy-efficiency with guaranteed exclusion of alarm states trips of equipment of TTLs, is in detail described in this article. Control algorithm was fine-tuned during procedure of simulation modelling. SAC with commutating structure passed field tests at elevator. Dynamics of control processes is examined.

Ключевые слова

Перегрузка зерна, поточно-транспортные линии, производительность, энергоэффективность, безаварийность, оптимизация, алгоритм, система автоматического управления, коммутируемая структура.

\section{Постановка задачи}

Процессы перегрузки зерна составляют основную часть технологических процессов предприятий (элеваторов), обеспечивающих его приемку и размещение в емкости для хранения, хранение и подработку (сушку, вентилирование, очистку), формирование коммерческих партий и отгрузку. У зерновых терминалов, обеспечивающих формирование крупных партий зерна и их отгрузку на железнодорожный, речной и морской транспорт, доля таких процессов максимальна. Процессы перегрузки реализуются поточно-транспортными линиями (ПТЛ), которые состоят из нескольких последовательно включенных конвейеров различных типов, обязательно включая в себя ковшовый конвейер для вертикального транспортирования - норию. Время выполнения операции перегрузки, полные и удельные энергозатраты на нее определяются производительностью ПТЛ. Целенаправленное изменение производительности ПТЛ осуществляется изменением степени открытия регулирующей задвижки и, следовательно, расхода зерна через нее из расходной емкости на первый, по ходу продукта, конвейер ПТЛ, т.е. ее загрузкой зерном. Увеличение загрузки сокращает время перегрузки и затраты энергии на ее реализацию, что, в конечном итоге, повышает экономическую эффективность работы предприятия.

Максимально достижимая производительность ПТЛ соответствует ее максимально допустимой загрузке, которая зависит от особенностей конструкции и эксплуатации конвейеров ПТЛ. Так, конструкция скребковых конвейеров и норий такова, что превышение их допустимой загрузки приводит к возникновениям завалов их рабочих объемов зерном (возникновения «подпора» зерна), [1]. Возникновение подпора означает возникновение аварийной ситуации (АС), когда дальнейший процесс перегрузки становится невозможен из-за угрозы перерастания АС в аварию. Такие угрозы ликвидируют специальные устройства аварийной защиты, отключающие конвейер, где возникла $\mathrm{AC}$, и все предшествующие ему конвейеры в аварийном режиме, т.е. без их разгрузки от транспортируемого материала. Отказы такой защиты, например, для нории, приводят к следующим негативным последствиям: а) развитию многотонных завалов зерна в точке его перегрузки с конвейера в башмак нории; б) заклиниванию ленты нории зерном, ее проскальзыванию на приводном барабане, перегреву от трения о приводной барабан, возгоранию, обрыву и обрушению ленты в норийные трубы, и, поскольку зерно имеет органическое 


\section{АВТОМАТИЧНІ І АВТОМАТИЗОВАНІ СИСТЕМИ УПРАВЛІННЯ ТЕХНОЛОГІЧНИМИ ПРОЦЕСАМИ}

происхождение, то ко взрыву воздушно-пылевой смеси.

Для управления процессом загрузки ПТЛ важно, что подпор зерна в башмаке нории или в коробе скребкового конвейера возникает через некоторое время после того, когда объемная производительность этих конвейеров стала меньше объемной производительности конвейеров, подающих в них зерно. Так, для нории, такая ситуация возникает, когда достигнут и превышен предел заполнения ее ковшей зерном, и, следовательно, достигнуто и превышено критическое значение объемной производительности нории. Важно, что это критическое значение априори неизвестно, т.к. на его конкретное значение влияют многие факторы, которые могут существенно изменяться непосредственно в ходе процесса перегрузки. К таким факторам относятся изменения характеристик: а) зерна, например, его фракционного состава, коэффициента внутреннего трения и т.д.; б) технического состояния нории, например, количества частично или полностью оборванных ковшей, степени натяжения и перекоса норийной ленты. Последние характеристики влияют на амплитуду и частоту колебаний ковшей и, следовательно, на фактическую степень их заполнения зерном.

В [2, 3] предложена концепция автоматического управления загрузкой ПТЛ, которая предполагает возможность обеспечить ее работу в окрестности максимально достижимой производительности, и, одновременно, предотвращения ее аварийных отключений из-за возникновения подпоров зерна в башмаке нории. В настоящей статье, на конкретном примере, рассмотрим вариант реализации САУ с коммутируемой структурой (САУ КС), которая реализует эту концепцию.

Особенности примера ПТЛ и блок-схема ее САУ КС

В качестве примера рассмотрим ПТЛ отгрузки зерна на железнодорожный транспорт из сушильно-складского комплекса емкостью 20 тыс. тонн в г. Хмельник (Винницкая область), см. рис. 1.

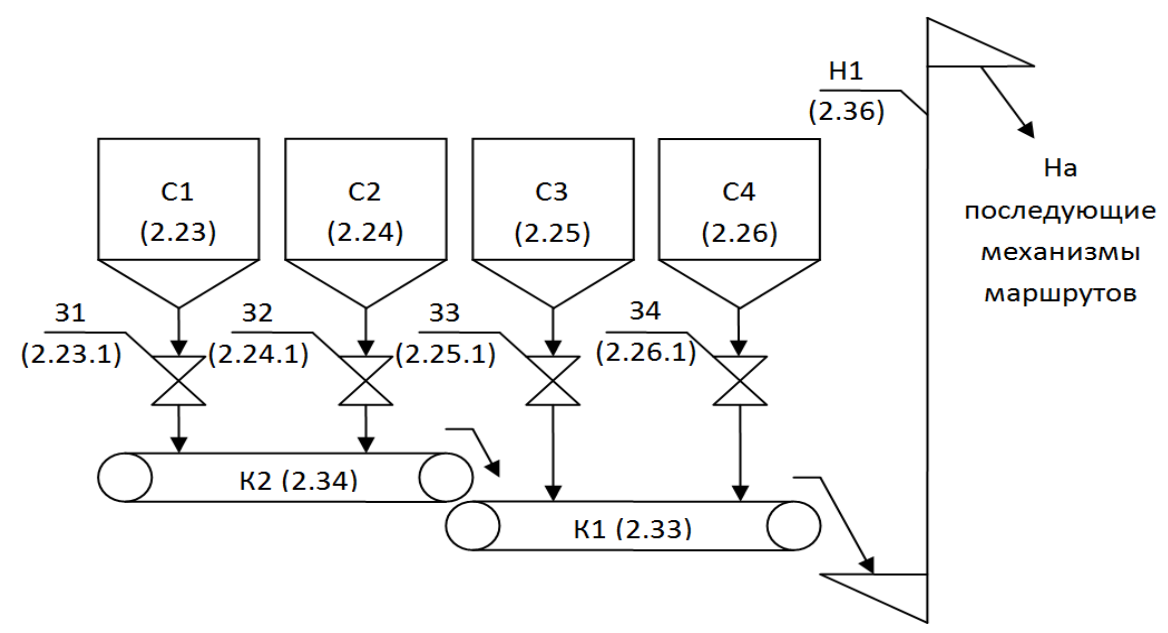

Рис. 1 - Упрощенная схема ПТЛ отгрузки зерна

C1-С4 - силоса для хранения зерна; 31-34-подсилоснье задвижки с электроприводом;

К1 и К2 -ленточные конвейеры с номинальной производительностью $100 \mathrm{~m} / \mathrm{v}$

(по пшенице насыпной массой $0,75 \mathrm{~m} / \mathrm{s}^{3}$ ), скорости лент 2,14 м/с, длины К1 - 79 м и К2 - 41 м;

H1 - нория с номинальной производительностью $100 \mathrm{~m} /$, скорость ленты 2,86 м/с, высота $25 \mathrm{M}$

Блок-схема варианта САУ КС загрузкой ПТЛ по рис. 1, к которой приводит реализация сформулированной концепции, приведена на рис. 2. 


\section{АВТОМАТИЧНІ І АВТОМАТИЗОВАНІ СИСТЕМИ УПРАВЛІННЯ ТЕХНОЛОГІЧНИМИ ПРОЦЕСАМИ}

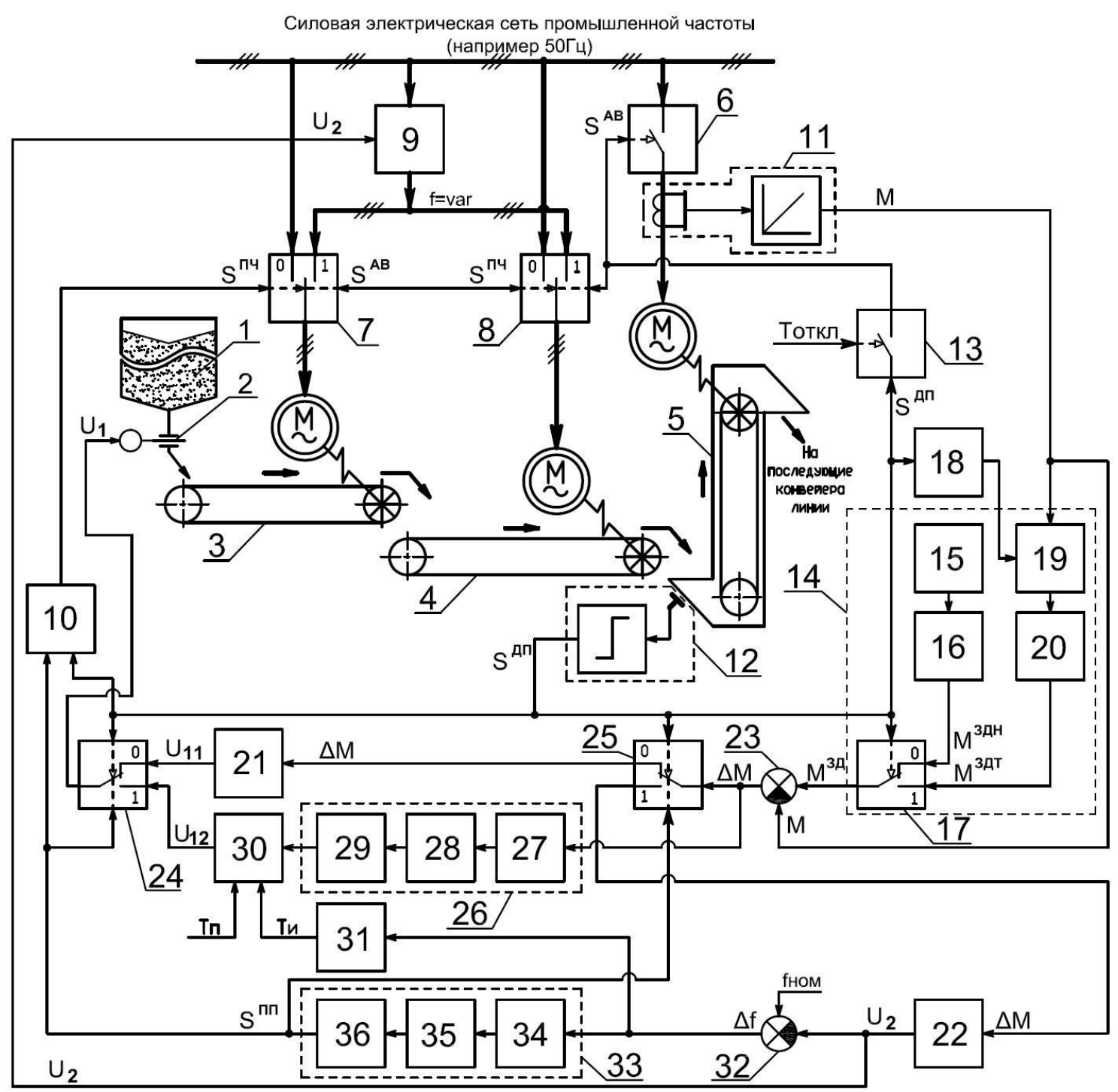

Рис. 2 - Блок-схема САУ КС загрузкой ПТЛ по рис. 1, реализующая сформулированную концепцию управления

Участок ПТЛ включает выбранный для отгрузки зерна силос 1, задвижку с исполнительным механизмом 2 , изменяющей расход материала в ПТЛ пропорционально сигналу управления $U_{1}$, два ленточных конвейера 3 и 4 , норию 5 с приводными электродвигателями (ПЭД) всех конвейеров. При любых вариантах систем непосредственное управление ПЭД осуществляется силовыми коммутационными устройствами 6,7 и 8, контроль нагрузки ПЭД нории - датчиком 11, а контроль подпора зерна в башмаке нории - датчиком 12 с задержкой на срабатывание (таймером) 13. Отметим, что цепи управления силовыми коммутационными устройствами, реализующие типовые функции включения и отключения электродвигателей при пуске и останове ПТЛ, и, непосредственно не связанные с реализацией алгоритмов управления САУ, на схеме не показаны.

В разработанной САУ для управления скоростью ПЭД конвейеров 3 и 4 дополнительно введен преобразователь частоты 9, изменяющий частоту пропорционально сигналу управления $\mathrm{U}_{2}$. Отметим, что все остальные блоки, реализующие разработанную САУ КС, виртуальные, т.к. реализуются в управляющем контроллере программным путем. К ним относятся логический элемент «ИЛИ» 10, задатчик нагрузки ПЭД нории 14, блок выбора кода транспортируемого материала 15, блоки хранения начальных (номинальных) значений нагрузки ПЭД нории 16, коммутатор заданного значения нагрузки ПЭД нории 17, формирователь импульса 18 , блок записи и хранения 19 , блок масштабирования 20, регуляторы нагрузки ПЭД нории 21 и 22 , алгебраические сумматоры 23 и 32, коммутаторы контуров регулирования 24 и 25, блоки оценки окончания переходного процесса 


\section{АВТОМАТИЧНІ І АВТОМАТИЗОВАНІ СИСТЕМИ УПРАВЛІННЯ ТЕХНОЛОГІЧНИМИ ПРОЦЕСАМИ}

26 и 33, блоки алгебраического модуля 27 и 34, фильтры низкой частоты 28 и 35, компараторы 29 и 36, генератор импульсов 30, блок алгебраического преобразования 31.

Описание алгоритма управления САУ на содержательном уровне

Отметим, что ПЭД нории 5 во всех режимах работы питается через блок 6 от силовой электрической сети промышленной частоты. ПЭД конвейеров 3 и 4 в номинальных установившихся режимах работы питаются от той же электрической сети. В этих режимах их коммутационные устройства 7 и 8 , по команде управления $\mathrm{S}^{\text {Пч }}=0$, включены в положение «0», а скорости ПЭД конвейеров и нории и, следовательно, их рабочих органов, перемещающих зерно, будут иметь постоянные номинальные значения. По команде управления $\mathrm{S}^{\text {Пч }}=1$ коммутационные устройства 7 и 8 переключаются в положение «1», и ПЭД конвейеров 3 и 4 переключаются на питание от преобразователя частоты 9, т.е. от специальной (локальной) электрической сети переменной частоты. При такой схеме питания скорости ПЭД конвейеров и, следовательно, их рабочих органов, будут изменяться пропорционально значению частоты на преобразователя частоты 9. Значение этой частоты, в свою очередь, будет задаваться сигналом управления $\mathrm{U}_{2}$, поступающим на управляющий вход частотного преобразователя 9. Команда управления $\mathrm{S}^{\Pi ч}$ включением преобразователя частоты 9 формируется логическим элементом «ИЛИ» 10 , как логическая сумма сигналов его входных сигналов $S^{\text {дП }}$ и $S^{\Pi \Pi}$, сущность которых описана ниже.

Текущая степень загрузки нории 5, а значит и всей ПТЛ, контролируется двумя датчиками. Во-первых, датчиком нагрузки ПЭД нории 11 с непрерывным выходным сигналом М, который измеряет величину, пропорциональную электрической мощности, потребляемой электродвигателем нории на перемещение ее рабочего органа из сети. Во-вторых, датчиком подпора зерна 12 с релейным выходным сигналом S дП, который контролирует предельно допустимый (критический) уровень заполнения башмака нории. Срабатывание датчика подпора, т.е. переход его выходного сигнала из состояния «0», когда $\mathrm{S}^{\text {ДП }}=0$, в состояние «1», когда $\mathrm{S}^{\text {дП }}=1$, означает, что степень заполнения башмака нории зерном достигла критического значения (событие $\left.\mathrm{S}^{\text {дП }}=1\right)$. Сигнал о событии

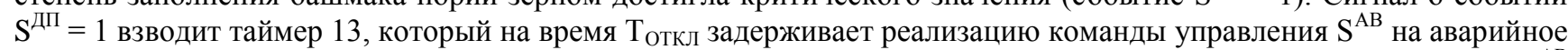
отключение ПЭД конвейеров от сети, т.е. на прекращение работы всей ПТЛ в аварийном режиме. Команда $\mathrm{S}^{\mathrm{AB}}$

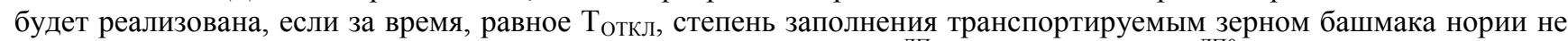

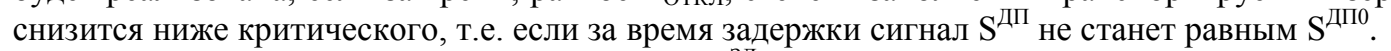

Заданное значение нагрузки ПЭД нории $\mathrm{M}^{3 Д}$ и, значит, всей ПТЛ формируется задатчиком 14 , который имеет два канала. Первый канал формирует начальное значение $\mathrm{M}^{3 д н}$, рассчитываемое с учетом типа и номинальных характеристик зерна этого типа и номинальной производительности нории на этом типе зерна. В блоке 15 хранятся варианты априори рассчитанных номинальных значений нагрузки ПЭД нории для транспортируемого зерна всех типов, исходя из выбранных условий, например, условия максимальной близости их к предельно допустимому объему заполнения ковшей нории. По коду материала, который задается в блоке 15, в блоке 16 выбирается соответствующее ему начальное заданное значение нагрузки ПЭД нории $\mathrm{M}^{3 Д н}$, и на вход коммутатора 17 подается «0». Второй канал формирует текущее заданное значение $\mathrm{M}^{3 д т}$, близкое к максимально достижимой

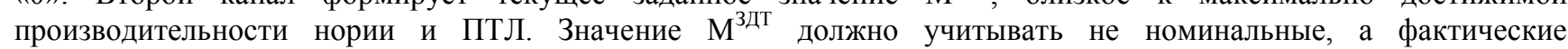
характеристики транспортируемого зерна и фактическую предельно достижимую производительность нории на этом типе зерна. Принципиально важно, что значения указанных переменных априори неизвестны и они могут существенно изменяться даже в процессе транспортирования одного типа зерна. Поэтому текущее значение $\mathrm{M}^{3 д т ~}$ может быть определено только в ходе самого процесса транспортирования.

Определение значения $\mathrm{M}^{3 Д т}$ происходит следующим образом. В момент срабатывания датчика подпора 12 ,

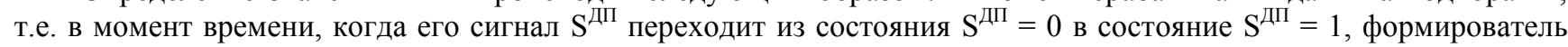
импульса 18 дает команду блоку записи-хранения 19 на запись и последующее хранение текущего значения нагрузки М ПЭД нории. Это значение соответствует критической нагрузке $\mathrm{M}^{\mathrm{KP}}$ ПЭД нории, т.е. нагрузке ПЭД нории в момент срабатывания датчика 12 , когда значение $\mathrm{M}=\mathrm{M}^{\mathrm{KP}}$. Значение $\mathrm{M}^{3 Д}$ рассчитывается в блоке масштабирования 20, где значение $\mathrm{M}^{\text {КР }}$ уменьшается до «докритического» уровня умножением $\mathrm{M}^{\mathrm{KP}}$ на

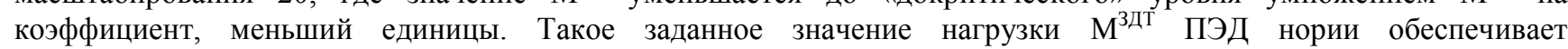
производительность нории, близкую к ее максимально достижимой производительности в конкретных условиях

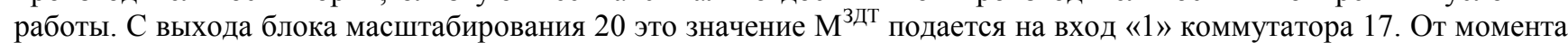
запуска ПТЛ в работу, когда $\mathrm{S}^{\text {дП }}=0$, до момента первого появления сигнала $\mathrm{S}^{\text {дП }}=1$, коммутатор 17 включен в состояние «0». Все это время на выходе задатчика 14 значение $\mathrm{M}^{3 д}$ будет равно начальному заданному значению нагрузки $\mathrm{M}^{3 д н}$. С момента первого появления сигнала $\mathrm{S}^{\text {дп }}=1$ коммутатор 17 переключается в состояние «1» и остается в этом состоянии вплоть до останова ПТЛ.

Стабилизация нагрузки М ПЭД нории 5 осуществляется одним из двух контуров стабилизации (регулирования). Для обоих контуров входными сигналами их регуляторов 21 и 22 является одна и также величина рассогласования между значениями $\mathrm{M}^{3 Д}$ и $\mathrm{M}$, которая вычисляется на сумматоре 23. 
Первый контур стабилизации реализуется через регулятор 21. Он меняет степень загрузки нории и ПТЛ зерном и, следовательно, нагрузки М ПЭД нории 5, изменяя положение задвижки 2 силоса 1 за счет изменения управляющего воздействия $\mathrm{U}_{1}=\mathrm{U}_{11}\left(\mathrm{U}_{11}\right.$ - это 1-й вариант формирования управляющего воздействия $\left.\mathrm{U}_{1}\right)$. Для работы этого (первого) контура коммутаторы 24 и 25 должны находиться в состоянии «0». Это условие выполняется от момента запуска ПТЛ до появления события $\mathrm{S}^{\text {ДП }}=1$. Важной особенностью первого контура стабилизации М является большое транспортное запаздывание в изменении нагрузки М ПЭД нории при изменении управляющего воздействия $\mathrm{U}_{1}$. Это запаздывание равно времени транспортирования зерна конвейерами 3 и 4 , причем его величина значительно превышает время Т Откл. Из-за этого запаздывания регулятор 21 может с приемлемым качеством стабилизировать значение $\mathrm{M}$ на уровне $\mathrm{M}^{3 Д}$ и, в частности, может не допускать появления таких динамических отклонений $\mathrm{M}$ от $\mathrm{M}^{3 Д}$, при которых возникают события $\mathrm{S}^{\text {ДП }}=1$ только при определенных условиях. Эти условия состоят в следующем - любые изменения в процессе транспортирования материала, последствия которых должен компенсировать регулятор 21 при стабилизации $\mathrm{M}$ за счет изменения $\mathrm{U}_{1}$, должны быть медленными. К таким изменениям относятся: а) изменения характеристик материала, сопровождающиеся изменениями скорости истечения зерна из бункера 1 на транспортер 3, и изменения его объемной массы; б) изменения технического состояния нории, приводящие к изменению предельного заполнения ковшей нории

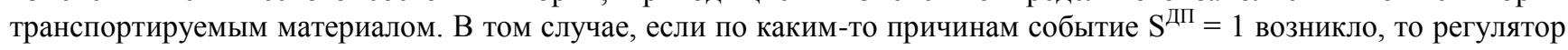
21 принципиально не способен устранить переполнение зерном башмака нории за время, меньшее или равное

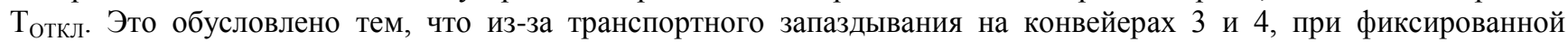
скорости их рабочих органов, уменьшение подачи зерна в норию 5 может начаться только через время, равное времени этого запаздывания.

Второй контур стабилизации М реализуется через регулятор 22. Он меняет степень загрузки нории и ПТЛ зерном и, следовательно, нагрузки М ПЭД нории 5 за счет изменения управляющего воздействия U 2 на преобразователь частоты 9, изменяя при этом частоту питания и, следовательно, частоту вращения ПЭД конвейеров 3 и 4 и, одновременно, скорость движения рабочих органов этих конвейеров. В этом, втором, контуре стабилизации транспортное запаздывание отсутствует, т.к. изменение скорости движения рабочих органов конвейеров и транспортируемого ими материала происходит практически одновременно по всей их длине. Это

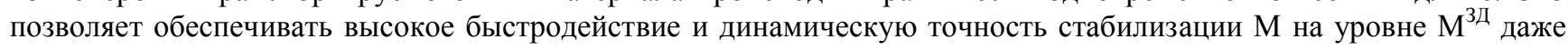
при больших скоростях изменения характеристик материала и технического состояния нории. Высокое быстродействие второго контура стабилизации $\mathrm{M}$ позволяет, даже в случае возникновения события $\mathrm{S}^{\text {ДП }}=1$, устранить его за время, меньшее, чем Т Откл, не допустив при этом аварийного отключения ПТЛ. Для работы этого (второго) контура коммутатор 24 может находиться в любом из состояний «0» или «1», а коммутатор 25 должен быть переключен в состояние «1». Исходя из удобства построения системы, целесообразно коммутатор 24 переключать в состояние «1» синхронно с коммутатором 25. Информацию об окончании процесса регулирования в этом контуре дает блок оценки окончания переходного процесса 26. Он анализирует значение разности заданной и фактической нагрузок $\left(\mathrm{M}^{3 Д}-\mathrm{M}\right)$ ПЭД нории 5, получаемую на выходе сумматора 23 и формирует команду в виде логического нуля («0»), если эти отклонения стали нулевыми. Блок 26 включает в себя следующие блоки: блок алгебраического модуля 27, делающего значения разности $\mathrm{M}^{3 Д}-\mathrm{M}$ знакопостоянными; фильтр низкой частоты 28 , который усредняет сигнал и предотвращает появление его нулевых значений при переходе через ноль разности $\mathrm{M}^{\text {зд }}-\mathrm{M}$; компаратор 29, формирующий из непрерывного логический (дискретный) сигнал ноль («0») или единица («1»). Сигнал логический «0» на выходе компаратора 29 и всего блока 26 появляется только тогда, когда условие $\mathrm{M}^{\text {зд }}-\mathrm{M}=0$ выполняется на таком по длительности интервале времени, на котором переходные процессы в фильтре низкой частоты 28 полностью закончатся, и сигнал на его выходе станет равным нулю. После окончания переходного процесса в этом контуре выходной сигнал датчика подпора зерна 12 гарантировано возвращается в состояние логического нуля $\left(\mathrm{S}^{\text {дП }}=0\right)$, и на первый вход логического элемента «ИЛИ» 10 будет поступать логический нуль. При этом, на его второй вход поступает логическая единица с блока окончания переходного процесса $33\left(\mathrm{~S}^{\Pi \Pi}=1\right)$. В силу выполнения логической функции «ИЛИ» на выходе логического элемента 10 сохраняется логическая единица $\left(\mathrm{S}^{\Pi ч}=1\right.$ - преобразователь 9 включен). Сигнал на выходе блока 33 будет равен логической единице $\left(S^{\Pi \Pi}=1\right)$ пока существует разность частот $\Delta f$, т.е. разность между частотой, которую генерирует преобразователь частоты 9, и частотой в силовой электрической сети. Эта разность поступает на вход блока 33 с выхода алгебраического сумматора 32. Блок 33 включает в себя: блок алгебраического модуля 34, фильтр низкой частоты 35 и компаратор 36. Блок 33 реализован и работает аналогично блоку 26.

Логический нуль на выходе блока 26 означает, что угроза возникновения события регулятором 22 ликвидирована, и может быть начата процедура вывода преобразователя частоты 9 из системы управления данной ПТЛ, в частности для получения возможности использования его в задачах управления другими ПТЛ. Поскольку действия регулятора 22 после возникновения события $\mathrm{S}^{\text {ДП }}=1$ всегда приводят к снижению скоростей ПЭД конвейеров 3 и 4 и скоростей движения их рабочих органов, то прямое переключение их питания от 
преобразователя частоты 9, дающего пониженную частоту, на общую сеть питания с промышленной частотой, привело бы к повторному событию $\mathrm{S}^{\text {дП }}=1$. Поэтому, перед таким переключением необходимо, при сохранении (стабилизации) нагрузки M ПЭД нории 5 на уровне найденного заданного значения $\mathrm{M}^{3 д т, ~ в ы в е с т и ~ к о н в е и ̆ е р ы ~} 3$ и 4 на скорость движения их рабочих органов, соответствующей номинальной частоте питающей сети. Для этого, при сохраняющемся положении коммутаторов 24 и 25 (оба включены в положение «1»), и, одновременно, при наличии логического «0» на выходе блока оценки окончания переходного процесса 26, генератор импульсов 30 выдает управляющий импульс $\mathrm{U}_{12}\left(\mathrm{U}_{12}-\right.$ это 2-й вариант формирования управляющего воздействия $\left.\mathrm{U}_{1}\right)$ на исполнительный механизм заслонки разгрузочного устройства 2 в направлении уменьшения его

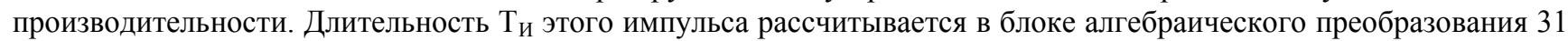

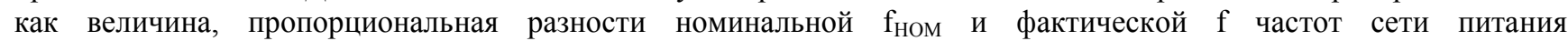
электродвигателей конвейеров ( $\left.\mathrm{f}_{\mathrm{HOM}}-\mathrm{f}=\Delta \mathrm{f}\right)$, полученной на выходе сумматора 32 . Это снижение производительности вызывает появление нового несоответствия между заданной $\mathrm{M}^{3 д т}$ и фактической М нагрузкой ПЭД нории. Устраняя это несоответствие, регулятор 22 будет повышать свое управляющее воздействие $\mathrm{U}_{2}$ и, следовательно, значение частоты для преобразователя частоты 9, повышая тем самым скорость ПЭД конвейеров. Если после первого импульса $\mathrm{U}_{1}=\mathrm{U}_{12}$, снизившего расход зерна через разгрузочное устройство 2 , величина

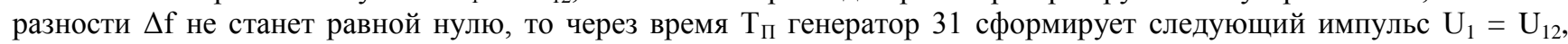
величина и знак которого будут зависеть от величины и знака разности $\Delta f$. Это, как и при первом импульсе, в конечном итоге, приведет к изменению частоты питания электродвигателей конвейеров. Подача импульсов $\mathrm{U}_{1}=$ $\mathrm{U}_{12}$ с интервалами времени $\mathrm{T}_{\Pi}$ будет продолжаться до тех пор, пока $\Delta \mathrm{f}$ не станет равной нулю. (На практике, для достижения условия $\Delta \mathrm{f}=0$, будет достаточно не более двух - трех импульсов). Время Т п должно быть выбрано равным или большим времени перемещения зерна конвейерами 3 и 4 от разгрузочного устройства 2 до его подачи в башмак нории 5 .

Информацию о полном завершении процесса подготовки к переключению ПЭД конвейеров к питанию непосредственно от сети, включая завершение переходных процессов в контуре стабилизации $\mathrm{M}$ изменением $\mathrm{U}_{2}$, дает блок оценки окончания переходного процесса 33. При достаточно продолжительном выполнении условия $\Delta \mathrm{f}$ $=0$, таком, что переходные процессы в фильтре 35 закончатся, и сигнал на его выходе станет нулевым, на выходе

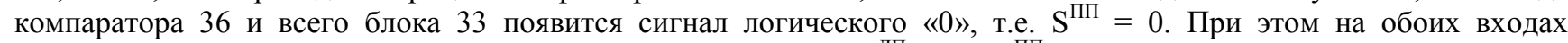
логического элемента «ИЛИ» 10 сигналы будут равны нулю $\left(\mathrm{S}^{\text {ДП }}=0\right.$ и $\left.\mathrm{S}^{\Pi \Pi}=0\right)$, что в соответствии с логической функцией «ИЛИ» сформирует на выходе этого логического элемента логический ноль $\left(\mathrm{S}^{\Pi ч}=0\right)$. По команде управления $\mathrm{S}^{\text {Пч }}=0$ коммутационные устройства 7 и 8 переключатся в положение «0», и ПЭД конвейеров начнут получать питание от общей сети питания. Одновременно с этим, коммутаторы 24 и 25 будут установлены в положение «0», замкнув контур стабилизации нагрузки М ПЭД нории через регулятор 21. На этом процедура вывода преобразователя частоты 9 из САУ ПТЛ, который был включен туда для ликвидации подпора зерна в башмаке нории, т.е. события $\mathrm{S}^{\text {ДП }}=1$, закончена. С этого момента преобразователь частоты 9 может быть использован в задачах управления другими ПТЛ, а рассматриваемая ПТЛ будет работать в режиме, близком к предельно достижимой производительности для текущих условий работы, которая будет поддерживаться регулятором 21. При повторном возникновении события $\mathrm{S}^{\text {ДП }}=1$ преобразователь частоты 9 вновь будет включен в систему управления ПТЛ до ликвидации события $\mathrm{S}^{\text {ДП }}=1$.

Способ управления загрузкой ПТЛ, вариант алгоритма которого был рассмотрен выше, запатентован, см. [4].

Результаты моделирования и производственных испытаний САУ КС. ФрагменТ моделирования САУ КС, детально иллюстрирующий описанный выше алгоритм управления, приведен на рис. 3. 


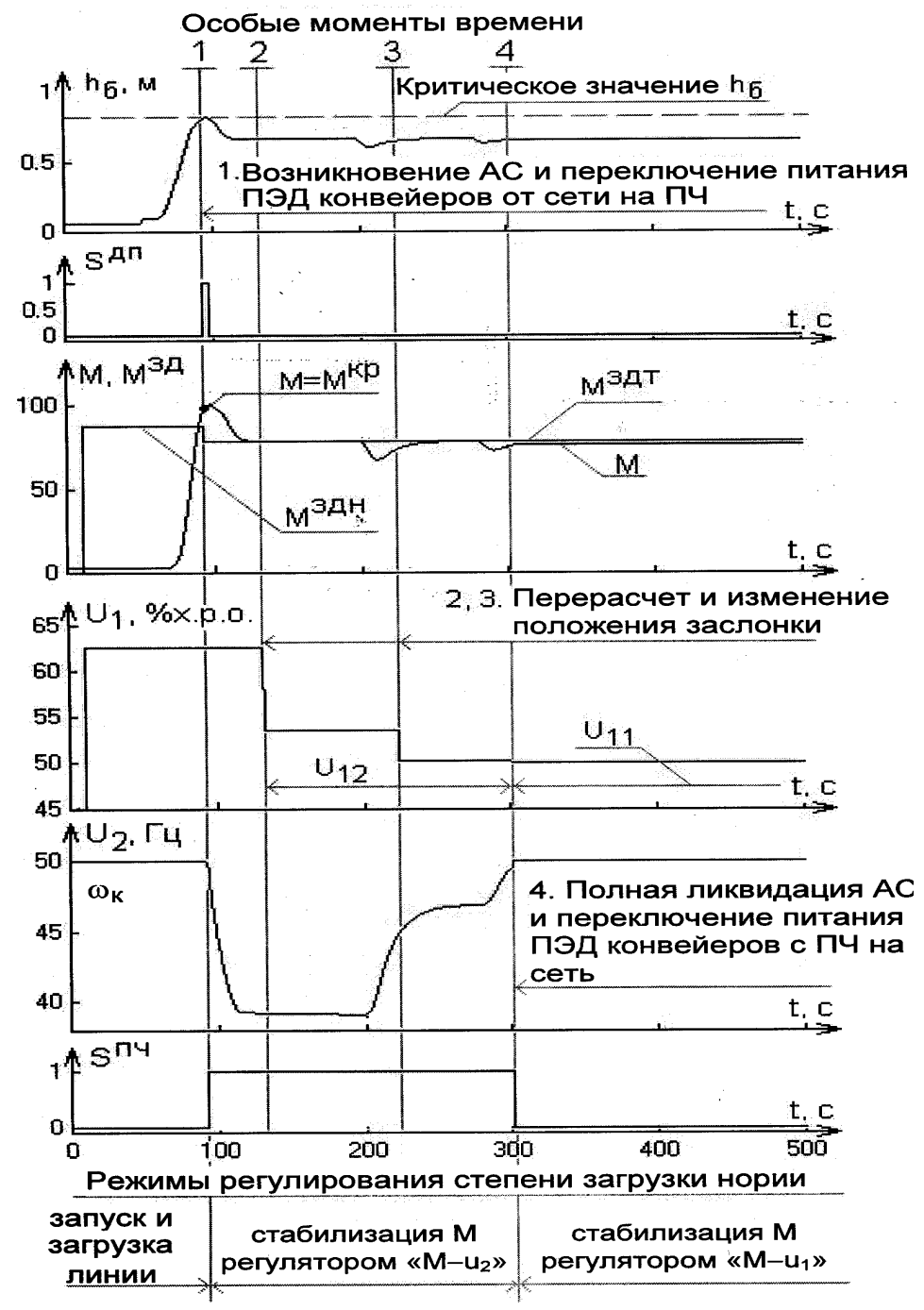

Рис. 3 - Динамика изменения переменных САУ КС на интервале от возникновения аварийной ситуации $\left(\mathrm{S}^{\text {ДП }}=1\right)$ до ее ликвидации $\left(\mathrm{h}_{\sigma}-\right.$ уровень зерна в башмаке нории)

Проанализируем результаты моделирования САУ КС и, прежде всего, коммутацию ее структуры. После запуска ПТЛ и «прокрутки» на холостом ходу, что гарантирует $\mathrm{S}^{\text {дП }}=0$, она была загружена зерном. Для этого САУ КС, установив значение $\mathrm{U}_{1}$, рассчитанное по $\mathrm{M}^{\text {здн }}$, открыла подсилосную задвижку до начального значения. Зерно, через время транспортирования конвейеров (время запаздывания), начинает поступать в башмак нории, что сопровождается ростом уровня $\mathrm{h}_{6}$ зерна в башмаке и момента сопротивления на валу М ее ПЭД. В момент времени «1» $\mathrm{h}_{б}$ достиг критического значения, что привело к срабатыванию датчика подпора $\left(\mathrm{S}^{\text {дП }}=1\right)$. Это событие означает, что начальная загрузка ПТЛ и ее нории, соответствующая $\mathrm{M}^{3 д н}$, оказалась выше критической. Синхронно со срабатыванием датчика подпора САУ КС: а) запоминает фактическое значение $\mathrm{M}^{\text {КР }}$ перерасчитывает по нему текущее заданное значение $\mathrm{M}^{3 д т}$; б) переключает питание ПЭД конвейеров с общей сети на питание от преобразователя частоты, $\mathrm{S}^{\Pi ч}=1$; в) переключает контур регулирования $\mathrm{M}$, т.е. «M - $\mathrm{U}_{1} », \mathrm{c}$ управляющего воздействия $\mathrm{U}_{1}$ на $\mathrm{U}_{2}$, (в контуре «M - $\mathrm{U}_{2}$ » нет транспортного запаздывания, что обеспечивает его высокие динамическую точность и быстродействие). Уменьшая $\mathrm{U}_{2}$ и скорость ПЭД конвейеров, САУ КС уменьшает подачу зерна на норию, обеспечивает $\mathrm{M}=\mathrm{M}^{3 д т}$ и предотвращает аварийный останов ПТЛ, см. момент времени «2». После окончания переходного процесса в этом контуре, см. момент времени «2», начинается процесс восстановления скорости ПЭД конвейеров до номинальной и освобождения преобразователя частоты. Для этого, в моменты времени «2», «3», «4» осуществляется перерасчет и изменение $\mathrm{U}_{1}=\mathrm{U}_{12}$. После окончания переходных процессов в контуре «M - $\mathrm{U}_{2} »$ этот контур размыкается, восстанавливается питание ПЭД конвейеров от сети, $\mathrm{S}^{\text {Пч }}=$ 0, и замыкается контур регулирования «M - $\mathrm{U}_{1} »$, который стабилизирует загрузку ПТЛ на уровне 


\section{АВТОМАТИЧНІ І АВТОМАТИЗОВАНІ СИСТЕМИ УПРАВЛІННЯ ТЕХНОЛОГІЧНИМИ ПРОЦЕСАМИ}

соответствующего $\mathrm{M}^{\text {Здт }}$.

Производственные испытания, см. [5], САУ КС были проведены на элеваторе в г. Хмельник при управлении загрузкой ПТЛ отгрузки зерна на железнодорожный транспорт, см. рис. 2. САУ КС была реализована в рамках разработанной и внедренной компанией С-инжиниринг АСУ ТП и получила название «автоматизированная система оптимизации загрузки» (АСО3). Испытания проводились в двух режимах: а) под управлением квалифицированного оператора; б) под управлением АСО3 (САУ КС). Результаты испытаний иллюстрируются фрагментами окон просмотра архива трендов переменных, см. рис. 4, функция которого предусмотрена в АРМ АСУ ТП. Окна отражают изменения переменных $\mathrm{M}$ и $\mathrm{U}_{1}$ - для варианта «а» и $\mathrm{M}, \mathrm{U}_{1}$ и $\mathrm{U}_{2}$ - для варианта «б» при управлении загрузкой ПТЛ. Верхний ряд окон соответствует условиям сильных колебаний истечения зерна из силоса, нижний ряд - в условиях умеренных колебаний. На рис. 4а, верхнем, видны два аварийных останова ПТЛ. На рис. 4 наличие коммутации структуры в САУ КС можно последить при отклонении $\mathrm{U}_{2}$ от $\mathrm{U}_{2}=50$.
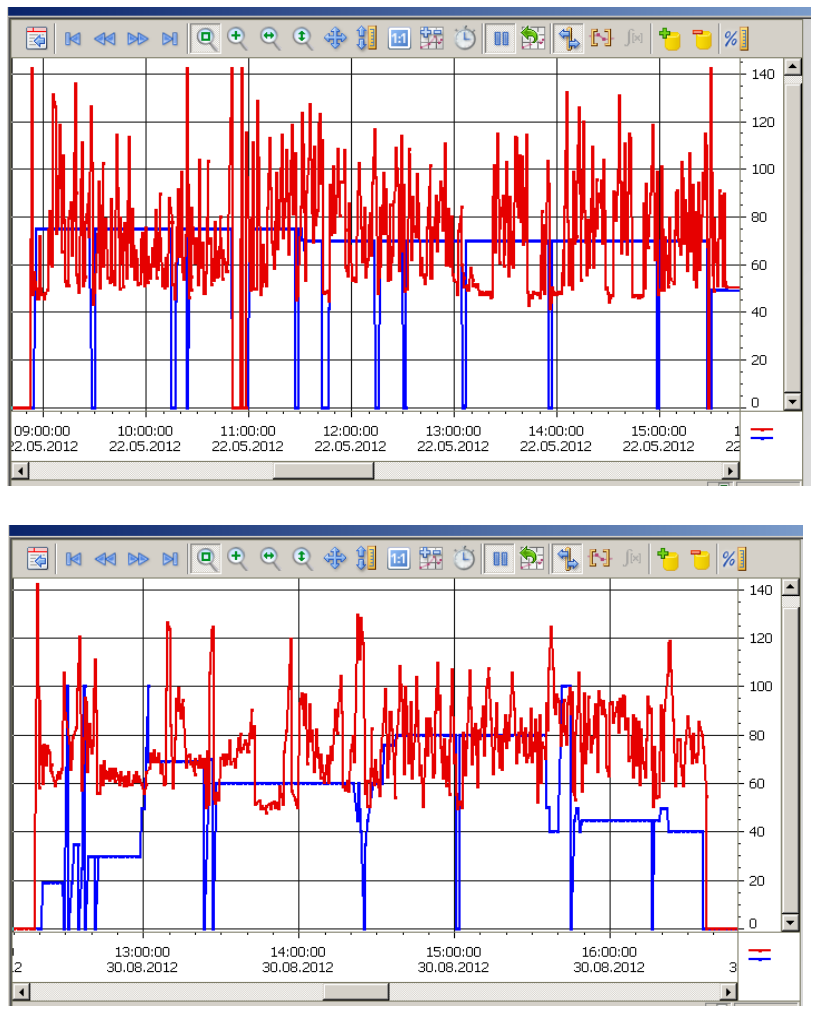

а) управление квалифицированным оператором
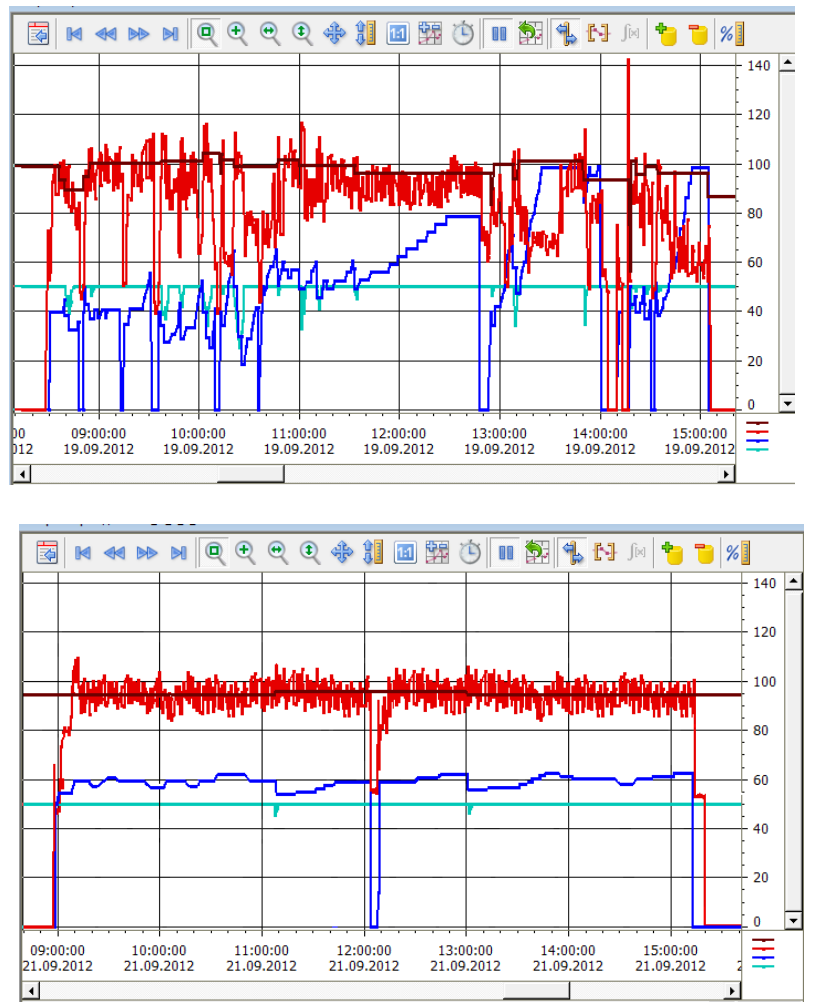

б) управление САУ КС

Рис. 4 - Динамика изменения нагрузки на ПЭД нории (M), положения регулирующей задвижки $\left(\mathrm{U}_{1}\right)$ и скорости (частоты) вращения ПЭД конвейеров $\left(\mathrm{U}_{2}\right)$ при различных вариантах управления загрузкой

\section{Заключение}

Автоматическое управление загрузкой ПТЛ, и, прежде всего, ее оптимизация, потенциально позволяет существенно повысить экономическую эффективность процессов перегрузки зерна на элеваторах. Создание эффективных САУ загрузкой осложняется следующими основными факторами: а) значение загрузки,

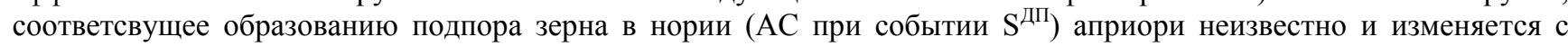
изменением механических свойств зерна и технического состояния нории; б) штатный канал регулирования загрузки изменением степени открытия подсилосной задвижки имеет очень большое транспортное запаздывание, что не позволяет ликвидировать возникающие подпоры.

Предложенная концепция автоматического управления производительностью ПТЛ и ее конкретизация в разработанной САУ КС дает возможность обеспечить работу ПТЛ в окрестности максимальной производительности, максимально быстро предотвращая развитие завала нории зерном, что гарантирует предотвращение аварийного отключения ПТЛ. 


\title{
АВТОМАТИЧНІ І АВТОМАТИЗОВАНІ СИСТЕМИ УПРАВЛІННЯ ТЕХНОЛОГІЧНИМИ ПРОЦЕСАМИ
}

\section{Литература}

1. Хобин В. А. Математическая модель нории как объекта управления с изменяющимися свойствами. Моделирование процесса заполнения ковшей / В. А. Хобин, И. Н. Кирьязов // Наук. пр. ОНАХТ. - Одеса: 2012. - Вип. 42. - Т. 1. - С. 358-368;

2. Хобин В. А. Концепция эффективного управления производительностью поточно-транспортных линий при перегрузке зерна / В. А. Хобин, С. В. Шестопалов // Хранение и переработка зерна. - Днепропетровск, 2011. - № 10 (148). - С. 26-29;

3. Шестопалов С. В. Оптимизация производительности процесса перегрузки зерна в условиях ограничения типа «аварийная ситуация» / С. В. Шестопалов, В. А. Хобин // XVIII Міжнарод. конф. 3 автомат. управління «Автоматика - 2011». - Львів, 2011. - С. 194-195;

4. Пат. на винахід 95887 Україна, МПК (2011.01), B65G 17/00, B65G 47/46 (2006.01), B65G 65/42 (2006.01), G01G 11/12 (2006.01). Спосіб автоматичного управління завантаженням потоково-транспортної лінії сипких матеріалів / Аннаєв Б. С., Герасимов В. В., Хобін В. А., Кір'язов І. М., Шестопалов С. В. і ін.; власник ТОВ «С-Інжинірінг». - № а201015861; заявл. 29.12.10; опубл. 12.09.11, Бюл. № 17. - 24 с;

5. Кирьязов И. Н. Автоматизированная система оптимизации загрузки поточно-транспортных линий перегрузки зерна: итоги производственных испытаний / И. Н. Кирьязов, С. В. Шестопалов // Хранение и переработка зерна. - Днепропетровск, 2013. - № 7 (172). - С. 43-46.

\section{References}

1. Hobin V. A. Matematicheskaya model norii kak ob'ekta upravleniya s izmenyayuschimisya svoystvami. Modelirovanie protsessa zapolneniya kovshey / V. A. Hobin, I. N. Kiryazov // Nauk. pr. ONAHT. - Odesa: 2012. - Vip. 42. - T. 1. - S. 358-368;

2. Hobin V. A. Kontseptsiya effektivnogo upravleniya proizvoditelnostyu potochno-transportnyih liniy pri peregruzke zerna / V. A. Hobin, S. V. Shestopalov // Hranenie i pererabotka zerna. - Dnepropetrovsk, 2011. - № 10 (148). - S. 26-29;

3. Shestopalov S. V. Optimizatsiya proizvoditelnosti protsessa peregruzki zerna v usloviyah ogranicheniya tipa «avariynaya situatsiya» / S. V. Shestopalov, V. A. Hobin // HVIII MIzhnarod. konf. z avtomat. upravlInnya «Avtomatika - 2011». - LvIv, 2011. - S. 194-195;

4. Pat. na vinahId 95887 UkraYina, MPK (2011.01), V65G 17/00, V65G 47/46 (2006.01), V65G 65/42 (2006.01), G01G 11/12 (2006.01). SposIb avtomatichnogo upravlInnya zavantazhennyam potokovo-transportnoYi lInIYi sipkih materIalIv / AnnaEv B. S., Gerasimov V. V., HobIn V. A., KIr'yazov I. M., Shestopalov S. V. I In.; vlasnik TOV «S-InzhinIrIng». - № a201015861; zayavl. 29.12.10; opubl. 12.09.11, Byul. № 17. - 24 s;

5. Kiryazov I. N. Avtomatizirovannaya sistema optimizatsii zagruzki potochno-transportnyih liniy peregruzki zerna: itogi proizvodstvennyih ispyitaniy / I. N. Kiryazov, S. V. Shestopalov // Hranenie i pererabotka zerna. Dnepropetrovsk, 2013. - № 7 (172). - S. 43-46.

\section{УСОВЕРШЕНСТВОВАННАЯ АСУ ТП ПЕРЕКЛЮЧЕНИЯ ПРОГРАММ РЕГУЛИРОВАНИЯ ЭНЕРГОБЛОКОМ.}

Improved PCS software switch control power generating

\author{
Плахотнюк А.А. ${ }^{1}$, Кокол Е.А. ${ }^{1}$, Максимов М.В. ${ }^{2}$ \\ Одесский национальный политехничный университет г.Одесса

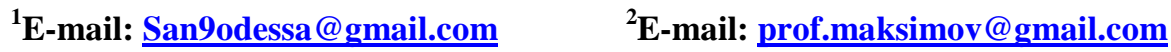

DOI: $10.15673 /$ 